\title{
Modelagem e Mobile Learning como ambiente para desenvolver conteúdos matemáticos e competências no Ensino Médio
}

\author{
Modeling and Mobile Learning as an environment to develop mathematical \\ content and competencies in High School
}

\author{
Neuber Silva Ferreira
}

Carlos Fernando Araújo Jr.

\begin{abstract}
Resumo: Neste artigo apresentamos uma pesquisa que teve como objetivo verificar quais contribuições podem ser observadas quando se utiliza situações-problema de Modelagem Matemática e dispositivos móveis (smartphones) como ambiente de aprendizagem para o ensino de conteúdos matemáticos, com 0 intuito de promover o conhecimento matemático e 0 desenvolvimento de competências e habilidades essenciais da Base Nacional Comum Curricular. A estratégia utilizada baseou-se na realização de atividades de Modelagem Matemática tendo como suporte os dispositivos móveis. A metodologia de pesquisa foi a Design Based Research. Participaram da pesquisa duas turmas do curso técnico integrado de um Instituto Federal. Os resultados mostram que a utilização do smartphone e seus aplicativos para a realização de atividades de Modelagem Matemática possibilitaram incrementar a formação dos estudantes, contribuindo para o entendimento dos conteúdos e para o desenvolvimento de habilidades e competências essenciais à Matemática no primeiro ano do Ensino Médio.
\end{abstract}

Palavras-chave: Modelagem Matemática. Dispositivos Móveis. Mobile Learning. Competência Matemática. BNCC.

Abstract: In this article we present a research that aimed to verify which contributions can be observed when using problem situations of Mathematical Modeling and mobile devices (smartphones) as a learning environment for teaching mathematical content, in order to promote knowledge mathematics and the development of essential competencies and skills of the Common Base National Curriculum. The strategy used was based on performing Mathematical Modeling activities with the support of mobile devices. The research methodology was Design Based Research. Students from two classes of the integrated technical course of a Federal Institute participated in the research. The results show that the use of the smartphone and its applications for the execution of Mathematical Modeling activities made it possible to increase the training of students, contributing to the understanding of the contents and the development of skills and competencies essential to Mathematics in the first year of high school.

Keywords: Mathematical Modeling. Mobile Divices. Mobile Learning. Mathematical Competence. BNCC.

Neuber Silva Ferreira Doutorando em Ensino de Ciências e Matemática. Professor do Instituto Federal de Educação, Ciência e Tecnologia de Minas Gerais (IFMG), campus Ouro Preto. Minas Gerais, Brasil.

iD orcid.org/0000-0002-1588-4254 $\bowtie$ neuber.ferreira@ifmg.edu.br Carlos Fernando Araújo Jr. Doutor em Física. Professor do Programa de Pós-Graduação em Ensino de Ciências e Matemática da Universidade Cruzeiro do Sul. São Paulo, Brasil. orcid.org/0000-0001-8188-161X $\bowtie$ carlos.araujo@cruzeirodosul.edu.br

Recebido em 27/06/2020 Aceito em 26/07/2020 Publicado em 27/08/2020 


\section{Introdução}

No Brasil e no mundo, as preocupações em relação à educação escolar têm orientado diferentes ações visando incrementar a formação dos estudantes, tanto em disciplinas específicas quanto no que tange à preparação para o trabalho e cidadania. A pesquisa que ora apresentamos vai ao encontro destas inquietações.

Conforme ponderações de autores como Sá e Paixão (2015), é importante que os sistemas educacionais forneçam aos jovens experiências relevantes para o mundo do século XXI. Isso significa a introdução de novos domínios de aprendizado caracterizados por habilidades e competências transferíveis. Consequentemente, para que estes novos domínios fossem introduzidos, aconteceram diversas ações e orientações internacionais de reorganização curricular, tendo como base o enfoque no desenvolvimento de competências.

O Brasil, alinhado a estas preocupações educacionais, buscou também promover ações neste sentido. Dentre as realizadas, vale destacar a construção e promulgação da Base Nacional Comum Curricular (BNCC). Conforme este documento, as aprendizagens essenciais devem concorrer para assegurar aos estudantes o desenvolvimento de competências gerais, que consubstanciam, no âmbito pedagógico, os direitos de aprendizagem e de desenvolvimento, guiando o desenvolvimento escolar em todas as etapas da educação básica (BRASIL, 2017).

Este foco - desenvolvimento de competências - tem orientado a construção de currículos da maioria dos Estados e Municípios do Brasil e de diversos países. Tal enfoque também é adotado nas avaliações internacionais de diversas organizações, como, por exemplo, a Organização para a Cooperação e Desenvolvimento Econômico (OCDE), que coordena o Programa Internacional de Avaliação de Alunos (PISA) e a Organização das Nações Unidas para a Educação, a Ciência e a Cultura (UNESCO), que instituiu o Laboratório Latino-americano de Avaliação da Qualidade da Educação para a América Latina (LLECE).

As orientações das diferentes ações para incrementar os novos domínios de aprendizagem indicam que as decisões pedagógicas devem estar direcionadas para 0 desenvolvimento de competências. Isto está presente na BNCC por meio da

indicação clara do que os alunos devem "saber" (considerando a constituição de conhecimentos, habilidades, atitudes e valores) e, sobretudo, do que devem "saber fazer" (considerando a mobilização desses conhecimentos, habilidades, atitudes e valores para resolver demandas complexas da vida cotidiana, do pleno exercício da cidadania e do mundo do trabalho) (BRASIL, 2017, p. 13). 
Em relação à área de Matemática e suas Tecnologias, a BNCC referente ao Ensino Médio propõe a ampliação e o aprofundamento das aprendizagens essenciais desenvolvidas no Ensino Fundamental e em continuidade a essas aprendizagens, foca na construção de uma visão integrada da Matemática, ainda na perspectiva de sua aplicação à realidade.

Nas indicações do documento, os estudantes devem empregar conceitos, procedimentos e estratégias não apenas para resolver problemas, mas também para formulá-los, descrever dados, selecionar modelos matemáticos e desenvolver o pensamento computacional, por meio da utilização de diferentes recursos da área. A BNCC salienta que quando a realidade é a referência, é preciso levar em conta as vivências cotidianas dos estudantes do Ensino Médio, envolvidos em diferentes graus, dados por suas condições socioeconômicas, pelos avanços tecnológicos, pelas exigências do mercado de trabalho, pela potencialidade das mídias sociais, entre outros (BRASIL, 2017).

O debate sobre competências em Matemática também tem sido realizado pelos especialistas em políticas de ensino de Ciências e de Matemática da Unesco (UNESCO, 2016). 0 foco no desenvolvimento de conhecimentos e das competências matemáticas vem sendo discutido por ser uma característica central das avaliações do PISA (OCDE, 2006, 2013), nas quais as questões referentes ao uso, envolvimento, compreensão e utilidade da Matemática em relação às demandas da vida foram enfatizadas. No estudo do PISA (OCDE, 2006), o desenvolvimento de conhecimentos e competências matemáticas estão, de certa forma, relacionadas com o conceito de mathematical literacy que é

\footnotetext{
a capacidade de identificar e entender o papel que a matemática desempenha no mundo, fazer julgamentos bem fundamentados e utilizar a matemática e envolver-se com ela de forma que atenda às necessidades de sua vida como cidadão construtivo, consciente e reflexivo (OCDE 2006, p. 133).
}

De acordo com a publicação Os desafios do ensino de Matemática na Educação Básica (UNESCO, 2016), no programa PISA, "as habilidades matemáticas são definidas como o poder de agir com inteligência e de maneira adequada em situações que envolvem alguma forma de desafio matemático" (p. 18). Portanto, é preciso assegurar "o desenvolvimento de conhecimentos e das competências matemáticas necessárias para a integração e a participação ativa na sociedade, assim como para a adaptação às mudanças necessárias (UNESCO, 2016, p. 13).

No entendimento de vários pesquisadores, desenvolver competências é fundamental para a educação, sobretudo em relação à habilidade em lidar com a incerteza dos dados e com a 
confiabilidade das informações que são fundamentais para a tomada de decisões baseadas em evidências envolvendo ética, economia e dimensões ambientais. Neste cenário, a Matemática merece destaque por seu papel no desenvolvimento da capacidade dos estudantes de analisar, raciocinar e tomar decisões participando de debates construtivos sobre questões locais e globais.

Pensando neste contexto é que a Modelagem Matemática e o Mobile Learning se apresentam como abordagens que podem elevar a Matemática dentro da educação para o século XXI. Conforme podemos observar nas pesquisas de English (2015), ao afirmar que a Modelagem Matemática possibilita desenvolver, nos estudantes, habilidades para lidar com a incerteza dos dados e das informações, detectar variações, realizar previsões, documentar, argumentar e perceber que os modelos são gerados à luz da incerteza. Bem como nas pesquisas de Araújo Junior et al. (2019), que destacam o potencial do Mobile Learning para o aprimoramento de competências e habilidades específicas, em particular nos campos da Matemática.

Ademais, a Modelagem e o Mobile Learning compreendem importantes recursos de aprendizagem que facilitam diferentes formas de integração das disciplinas, além de envolver vários recursos que suportam esta integração, incluindo trabalhos com projetos, resolução de problemas, planejamento e realização de investigações, analisar e representar dados de várias maneiras bem como testar e desenvolver, aplicar e avaliar modelos baseados em evidências.

Sendo assim, buscando contribuir para uma Educação Matemática focada em ações que visam incrementar a instrução dos estudantes, tanto nos conteúdos matemáticos específicos, quanto no desenvolvimento de habilidades e competências essenciais à formação destes para 0 trabalho e cidadania, é que apresentamos como questão de investigação: Que contribuições podem ser observadas, quando se utilizam atividades de modelagem matemática e dispositivos móveis (smartphones), como ambiente de aprendizagem para o ensino de conteúdos matemáticos e para o desenvolvimento de competências e habilidades essenciais da BNCC?

Com o intuito de responder a esta questão, o presente artigo apresenta parte de uma pesquisa que investigou uma proposta de ensino utilizando atividades de modelagem matemática e dispositivos móveis como ambiente de aprendizagem. A Modelagem Matemática é uma tendência da Educação Matemática recomendada por educadores e nos documentos oficiais. De acordo com Pereira et al. (2017), esses documentos sinalizam que o trabalho com a Modelagem Matemática pode ser um meio para desenvolver competências e habilidades inerentes à formação do estudante. 
Neste sentido, as atividades desenvolvidas nesta pesquisa foram planejadas de forma a alinhar os objetivos e resultados de aprendizagem como maneira de fornecer dados com o intuito de analisar as contribuições para desenvolvimento das competências e habilidades (BNCC , 2017), do conhecimento matemático e da abordagem de conteúdos matemáticos em duas turmas do Ensino Médio.

Nas seções seguintes, iremos apresentar uma breve revisão do referencial teórico, Modelagem Matemática e Mobile Learning no ensino de Matemática, que fundamentaram a investigação; a metodologia de pesquisa empregada no trabalho; os resultados; e conclusões.

\section{Modelagem Matemática}

A Modelagem Matemática, de acordo com Blum e Ferri (2009), pode ser entendida como o processo de tradução entre o mundo real e a Matemática em ambas as direções. Suas raízes estão ancoradas na Matemática Aplicada, em que os "modeladores" a utilizam em processos de previsão, otimização e representação de fenômenos naturais, físicos, químicos, entre outros, por meio de modelos matemáticos (BASSANEZI, 2002).

No âmbito da Educação Matemática, a Modelagem Matemática pode ser entendida como "uma alternativa pedagógica na qual fazemos uma abordagem, por meio da Matemática, de uma situação-problema não essencialmente Matemática" (ALMEIDA, SILVA e VERTUAN, 2012, p. 17). D’Ambrósio (1986) salienta que a "modelagem é um processo muito rico de encarar situações e culmina com a solução efetiva do problema real e não com a simples resolução formal de um problema artificial" (p. 11). Para esse autor, fica evidente que o objetivo é promover o ensino da Matemática de modo que problemas fictícios sejam deixados de lado, já que muitas vezes estão desvinculados de problemas do dia a dia, e passem a trabalhar com modelos reais que fariam mais sentido de serem vistos em sala de aula.

Para Barbosa (2004), Ferreira (2013), Braga e Espírito Santo (2017), a Modelagem Matemática é entendida como um ambiente de aprendizagem. No entendimento dos dois últimos, a Modelagem Matemática, "ao buscar solução para uma situação problema advinda de temas de investigação, fazendo uso de modelos matemáticos, promove um ambiente de aprendizagem que como tal envolve alunos e professores no processo" (BRAGA e ESPÍRITO SANTO, 2017, p. 147). Nesta concepção, Barbosa (2004) apresenta a modelagem como um ambiente de problematização e investigação em que "o primeiro refere-se ao ato de criar perguntas e/ou 
problemas enquanto que o segundo, à busca, seleção, organização e manipulação de informações e reflexão sobre elas" (p. 4).

Sendo assim, o desenvolvimento de atividades de Modelagem Matemática coloca os estudantes "em um contexto de aprendizagem em que a discussão de situações-problema, a participação ativa e o uso de diferentes registros se fazem essenciais" (VERTUAN, 2007, p. 131). Esses argumentos estão relacionados, por um lado, com a motivação que as atividades podem proporcionar aos estudantes e com a aplicabilidade da Matemática; por outro lado, em um sentido mais amplo, com aspectos extramatemáticos, vinculados à competência crítica e reflexiva dos discentes.

No âmbito das salas de aula, diversas experiências de atividades de Modelagem Matemática têm sido conduzidas no Brasil e no exterior. Nessas práticas, o encaminhamento das atividades varia, muitas vezes, em função da necessidade de se atender às exigências impostas pelo contexto escolar e, também, de acordo com as concepções sobre o papel da modelagem no currículo.

Os procedimentos para a condução e compreensão da atividade de modelagem são, na maioria das vezes, apresentados em termos do processo de construção do modelo matemático', que se prestam a descrever determinada problemática e lhe sugerir soluções atuando de forma a orientar as ações. Este processo envolve um conjunto de atos desenvolvidos pelos estudantes experimentação, seleção de variáveis, formulação de hipóteses, simplificações, resolução de problemas e validação do modelo vinculado ao contexto de uma situação não essencialmente matemática (ALMEIDA, SILVA e VERTUAN, 2012)

Segundo Silva (2018), apesar de a modelagem ser uma tendência na Educação Matemática, na Educação Básica ela ainda é pouco utilizada e consequentemente, torna-se importante que os professores desenvolvam nos alunos a competência de Modelagem Matemática. Ademais, conforme Blum (2015), esta é uma atividade de demanda cognitiva, que impõe dificuldades aos estudantes, uma vez que várias competências estão envolvidas, que podem ser não matemáticas, conhecimento matemático e extramatemático, noções conceituais, bem como convicções e atitudes adequadas. De acordo com Blum e Leiß (2007), a competência

\footnotetext{
1 "Um modelo matemático consiste em um sistema conceitual, descritivo ou explicativo, expresso por meio de uma linguagem ou de uma estrutura matemática, com a finalidade de descrever o comportamento de outro sistema e permitir a realização de previsões sobre este outro" (ALMEIDA, SILVA e VERTUAN, 2012, p. 21).
} 
de Modelagem Matemática pode ser definida como

\begin{abstract}
a capacidade de identificar questões, variáveis, relações ou suposições relevantes em uma determinada situação do mundo real, traduzi-las em matemática e interpretar e validar a solução do problema matemático resultante em relação à dada situação, bem como a capacidade de analisar ou comparar determinados modelos, investigando as suposições feitas, verificando as propriedades e o escopo de um determinado modelo (p. 227).
\end{abstract}

Nesse sentido, Silva (2018) pondera que "quando lidamos com alunos inexperientes em Modelagem Matemática, há a necessidade de introduzir a modelagem de forma gradual" (p. 27). De acordo a autora, uma forma de fazer isso é por meio de momentos de familiarização com a Modelagem Matemática.

A familiarização é muito bem discutida no trabalho de Almeida e Dias (2004). Essas autoras propõem que as atividades de modelagem sejam apresentadas aos estudantes de forma gradativa, em "três momentos de familiarização". Em atividades do primeiro momento, o professor apresenta uma situação-problema, juntamente com outras informações necessárias e os estudantes, reunidos em grupo, investigam a possível solução. Nas atividades do segundo momento de familiarização, o professor pode sugerir uma situação-problema aos estudantes que, divididos em grupos, coletam mais informações para a investigação da situação. Assim, as atividades de modelagem do segundo momento se caracterizam por uma maior independência do estudante referente à definição de procedimentos para realizar a investigação. Durante atividades de modelagem do terceiro momento, os estudantes, também em grupos, são responsáveis pela condução do trabalho.

É importante salientar que participar de atividades de Modelagem Matemática possibilita aos estudantes o desenvolvimento de competências e habilidades que são importantes para seu crescimento como pessoa e para o mundo do trabalho. Uma vez que esta tem como princípios norteadores a observação da realidade, discussões e investigações de problemas por meio da Matemática. Ademais, por se tratar de uma investigação em sala de aula, as situações problemas são oriundas da realidade dos estudantes e de seus contextos sociais, da escola e de suas imediações (ZANELLA e KATO, 2017).

$\mathrm{Na}$ pesquisa que ora apresentamos, as atividades de Modelagem Matemática foram pensadas de forma a oportunizar aos estudantes os momentos de familiarização, pois não tiveram contato com a modelagem como ambiente de aprendizagem. Os dispositivos móveis foram 
utilizados para compor este ambiente, apoiando o desenvolvimento do trabalho, potencializando a aprendizagem e o desenvolvimento de competências e habilidades conforme preconiza a BNCC.

\section{Mobile Learning no ensino de Matemática}

Com desenvolvimento, proliferação e evolução das tecnologias, principalmente as móveis, muitas investigações têm sido realizadas para explorar a forma como podem ser melhor utilizadas nos processo de ensino e de aprendizagem (MOURA, 2010). Os trabalhos publicados sobre essas investigações relatam a importância desses recursos no processo de aprendizagem, alegando que muitas instituições de ensino estão implementando a aprendizagem móvel para oferecer flexibilidade ou mesmo para preparar estudantes e professores para a área digital em desenvolvimento, conforme ponderam Briz-Ponce et al. (2017).

Organizações governamentais como a Organização das Nações Unidas para a Educação, a Ciência e a Cultura, por meio da publicação O Futuro da aprendizagem móvel: implicações para planejadores e gestores de políticas (UNESCO, 2014) também reconhecem a relevância e têm recomendado às administrações públicas que adotem infraestrutura tecnológica para assegurar 0 acesso igual à conectividade móvel, a fim de permitir que os estudantes tenham disponíveis uma gama importante e crescente de possibilidades de aprendizagem.

Segundo Moura (2010), à medida que os campos da aprendizagem móvel e dos dispositivos móveis se desenvolvem, possibilitando novas formas de aprendizagem, surge 0 conceito de Mobile Learning, um eixo de investigação importante no domínio dos ambientes informatizados para a aprendizagem humana. Muito foi discutido sobre o conceito de Mobile Learning e são várias as definições existentes.

Crompton (2013) define Mobile Learning como "aprendizagem em múltiplos contextos, através de interações sociais e de conteúdo, usando dispositivos eletrônicos pessoais" (p. 48). No entendimento desta autora, a aprendizagem é isenta, livre de uma ligação, de partir de um local específico, bem como a forma como os estudantes podem aprender usando um dispositivo móvel para se conectar a pessoas e artefatos; torna este método de aprendizagem desprovido de restrições espaciais ou temporais e pode permitir que a aprendizagem ocorra em uma abordagem mais centrada no estudante.

Em relação ao ensino de Matemática, Crompton e Traxler (2015) afirmam que os 
dispositivos móveis estão apoiando os educadores matemáticos nas salas de aula em todo 0 mundo. Alguns atributos da tecnologia, como capacidades gráficas, fazem com que sua utilização seja considerada, como um aspecto positivo em facilitar a construção da compreensão em Matemática. De acordo com os autores, "usando a tecnologia, os alunos são capazes de construir cognitivamente redes de relacionamentos que ligam conceitos e processos matemáticos" (CROMPTON e TRAXLER, 2015, p. 1).

Quanto à utilização dos dispositivos móveis, Sawaya e Putnam (2015) afirmam que para os professores projetarem atividades de aprendizado móveis para conectar a Matemática dentro da escola com o mundo além da escola, eles precisam considerar a interação, a integração e a interdependência entre os objetivos de aprendizado da disciplina, as possibilidades dos dispositivos móveis e os tipos de atividades de aprendizado. Sendo assim, os professores deverão refletir se querem que os estudantes resolvam um problema, formem conexões ou usem representações matemáticas. Além disso, os professores precisam identificar qual aspecto do dispositivo móvel eles solicitarão aos estudantes para alcançar esses objetivos. Depois de avaliar o objetivo geral de aprendizagem e a(s) oportunidade(s) específica(s) do smartphone, os educadores podem decidir sobre o tipo de atividade de aprendizado móvel que desejam projetar, na tentativa de preencher a lacuna entre a Matemática escolar e a de fora da escola.

Neste contexto, o Mobile Learning se insere como uma alternativa que pode contribuir provendo experiências educacionais relevantes e fomentando 0 desenvolvimento das competências e habilidades desejadas. Conforme afirmam Araújo Junior et al. (2019), muitos estudiosos sugerem que os dispositivos móveis no paradigma do Mobile Learning podem ser utilizados para ampliar as pedagogias e desenvolver habilidades para o século XXI, porém poucos trabalhos os exploraram para o aprendizado contextual e situado. Eles também afirmam que desenvolvimentos recentes neste campo "apontaram a importância dessa modalidade para integrar um sistema de ensino e aprendizagem mais ativo e abrangente, que difunde os domínios da sala de aula convencional e práticas convencionais de ensino e aprendizagem" (ARAUJO JUNIOR et al., 2019, p. 182).

Finalizamos as considerações sobre Mobile Learning afirmando nossa crença de que este recurso, além de desenvolver competências e habilidades relacionadas ao desenvolvimento tecnológico, intrínseca à tecnologia móvel, também pode contribuir em relação aos outros conhecimentos relacionados aos processos de ensino e de aprendizagem de Matemática, 
promovendo a equidade digital, proporcionando experiências capazes de quebrar barreiras entre os ambientes de aprendizagem formal e informal e propiciando aos estudantes a possibilidade de ter acesso às informações de uma forma rápida, em qualquer lugar ou momento.

\section{Metodologia de pesquisa}

A pesquisa foi orientada metodologicamente tendo como referência a Design Based Research (DBR). A escolha aconteceu em função de suas características, que estão relacionadas com a fundamentação na teoria e na prática, na análise da literatura e de casos, em métodos qualitativos e quantitativos (PLOMP e NIEVEEN, 2013).

No campo da Educação, esta metodologia vem se destacando e se consolidando nas investigações sobre uso de tecnologias. A DBR assume como fundamento a integração entre a pesquisa e o desenvolvimento de intervenções educativas em contextos reais de aprendizagem, oferecendo uma lente para investigar e compreender como, quando e porque uma inovação educacional funciona na prática (RAMOS, GIANNELLA e STRUCHINER, 2010).

É importante salientar que, nesta pesquisa, buscamos promover a melhoria das práticas educativas ao verificar como um ambiente de aprendizagem que utiliza a Modelagem Matemática e o Mobile Learning, que são tendências educacionais já consolidadas na literatura, pode contribuir para os processos de ensino e de aprendizagem da Matemática no Ensino Médio. Sendo assim, implementar, analisar e avaliar este ambiente se insere numa pesquisa na metodologia DBR.

Entendemos também que essas características são importantes para a investigação por estarem relacionadas com o ensino e aprendizagem, em que é necessária a observação contínua de aspectos qualitativos associados a repetição e avaliação de processos. Além do mais, conforme afirmam Anderson e Shattuck (2012) apud Nunes (2019), a metodologia DBR foi projetada por e para educadores com o objetivo de aumentar o impacto, a transferência e a tradução da pesquisa em Educação para melhorar a prática. Isto é o que se pretende com esta investigação: pesquisar para aprimorar a experiência relacionada à aprendizagem de Matemática por meio de atividades de Modelagem Matemática e da utilização de dispositivos móveis.

A pesquisa foi realizada no Instituto Federal de Educação, Ciência e Tecnologia de Minas Gerais (IFMG), campus Ouro Preto, uma vez que, o professor pesquisador faz parte do corpo docente da instituição e busca, com sua pesquisa, contribuir para melhorar a proficiência dos 
alunos em Matemática.

Os dados foram coletados durante as aulas e nos momentos de interação virtual com os dispositivos móveis, durante duas semanas, em duas turmas de $1^{0}$ ano do curso Técnico Integrado em Mineração.

Os participantes do estudo foram todos os envolvidos na pesquisa, ou seja, alunos, professor e pesquisador. Foram, ao todo, 80 estudantes, com idade média de 15 anos, inicialmente matriculados nestas duas turmas, os quais concordaram em participar da pesquisa e assinaram termo de consentimento livre e esclarecido2. O professor participou na elaboração, no desenvolvimento e na avaliação das atividades; já o pesquisador, das três primeiras etapas e da análise das atividades.

Os instrumentos de coleta de dados utilizados foram: caderno de campo; gravações em áudio e vídeo de algumas aulas e das apresentações dos trabalhos; questionário online; trabalhos entregues durantes as aulas; notas de aula do professor regente e todas as informações compartilhados pelos estudantes nos seus dispositivos móveis em grupos de WhatsApp, no YouTube, aplicativos educacionais, dentre outros. A utilização de todos estes instrumentos de coleta de dados justifica-se pela metodologia de pesquisa adotada.

Neste estudo vamos relatar o desenvolvimento de uma das quatro atividades de Modelagem Matemática que ocorreram durante os dois primeiros trimestres do ano letivo de 2019. A atividade aqui relatada foi planejada para contextualizar os conceitos relacionados às funções quadráticas e teve como tema as "Parábolas na Arquitetura".

Como mencionado anteriormente, a atividade foi desenvolvida em duas turmas do primeiro ano do curso Técnico Integrado em Mineração e teve, em média, duração de quatro aulas. Além dos momentos presencias, acorreram também os de aprendizado com mobilidade, Mobile Learning, por meio da utilização dos smartphones e seus aplicativos. Estes momentos possibilitaram coleta de informações, fomentar discussões, compartilhar resultados de investigações e expor as reflexões.

\footnotetext{
${ }_{2}^{2}$ Projeto de pesquisa apresentado ao Comitê de Ética em Pesquisa e aceito em 20 de dezembro de 2018 - Número do parecer: 3.098.527.
} 


\section{Descrição das atividades desenvolvidas e dos procedimentos realizados}

Em ambas as turmas os procedimentos foram similares. No início da abordagem, os estudantes foram informados sobre a atividade e quais os objetivos de sua aplicação. Em seguida, foi solicitado que se agrupassem para que as orientações fossem distribuídas. Depois, realizouse uma breve explicação sobre o que era esperado dos estudantes em relação à prática. Durante a realização da atividade, o professor e o pesquisador participaram como mediadores, auxiliandoos em relação ao entendimento do problema e estimulando-os a refletirem sobre o que deveriam investigar.

Como os estudantes já haviam realizado uma atividade nos moldes do primeiro momento de familiarização, a atividade Parábolas na Arquitetura foi proposta em conformidade com 0 segundo momento de familiarização da modelagem (ALMEIDA e DIAS, 2004). Neste caso, 0 professor sugeriu uma situação-problema aos estudantes que, divididos em grupos, coletaram mais informações para a investigação da situação. A atividade teve como objetivo abordar de forma investigativa os principais conceitos de função quadrática por meio de relações estabelecidas entre a visualização gráfica e os conceitos algébricos, bem como estimular os estudantes a investigar situações que os conduziriam aos conceitos matemáticos relacionados com a função quadrática. Tais conceitos foram obtidos por meio da manipulação e observação de imagens e de gráficos gerados pelo aplicativo GeoGebra.

Esta forma de abordar e desenvolver este tipo de função é sugerida por diversos autores, como Maia (2007), Pádua (2010), Bolzan, Flores e Gois (2014) e Heinen (2015), os quais salientam que se tornou necessário trabalhar com novas abordagens por causa da ênfase dada para 0 ensino de funções, na maioria das vezes, por via da álgebra, sendo comum nos livros didáticos maior destaque para a expressão analítica e pouco para os aspectos gráficos ou tabulares, conforme afirmam Borba e Penteado (2010). De acordo com esses autores, "é difícil a geração de diversos gráficos num ambiente em que predomina o uso de lápis e papel e, então, faz sentido que não se dê muita ênfase a esse tipo de representação" (BORBA e PENTEADO, 2010, p. 31).

Sendo assim, os autores mencionados recomendam utilizar estratégias que não privilegiem apenas um tipo de representação, sendo importante desenvolver outras também, como a expressão algébrica, o gráfico e a tabela. No entendimento deles, "conhecer sobre funções passa a significar saber coordenar representações" (BORBA e PENTEADO, 2010, p. 32).

Tendo como referência os trabalhos desses autores, julgamos ser pertinente para 0 
ambiente de aprendizagem uma abordagem que utilizasse investigação, estudo, análise e construção de gráficos da função quadrática. É importante destacar que algumas definições e conceitos relacionados com as funções quadráticas foram inicialmente abordados e trabalhados nas aulas de forma tradicional e determinadas dificuldades foram detectadas pelo professor como por exemplo, associar o gráfico da função com sua expressão algébrica.

A atividade então foi projetada para ser realizada em três etapas. A primeira consistia no reconhecimento da curva (parábola) em imagens existentes na Arquitetura e no cotidiano dos alunos. Segundo Machado (2007), "não tem como ignorar a presença das parábolas no dia-a-dia, portanto, é necessário que se dê o seu devido valor, mostrando aplicações quando forem abordadas durante as aulas" (p. 9).
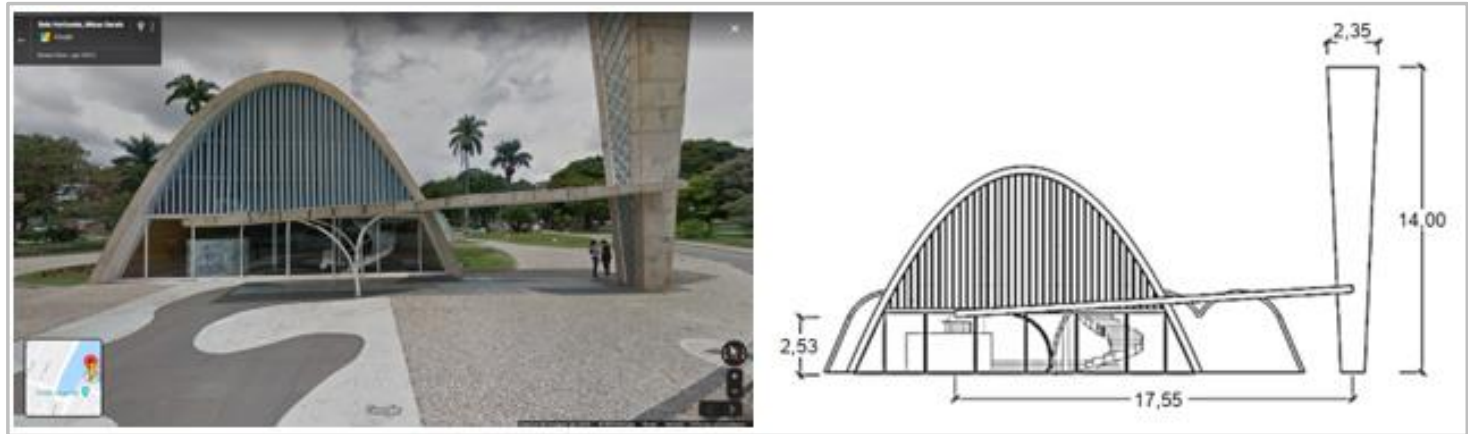

Figura 1: Igreja da Pampulha em Belo Horizonte (Google imagens $\left.{ }^{3}\right)$

Seguindo este entendimento, foi solicitado aos estudantes que procurassem por imagens na internet, utilizando seus smartphones, e apresentassem as figuras escolhidas aos demais colegas de turma. Foram encontradas imagens de construções, obras de arte, equipamentos, dentre outras. Durante a realização da atividade, os estudantes enviaram as figuras escolhidas, utilizando o aplicativo WhatsApp, ao grupo da sala e o professor projetava para que todos pudessem observá-las. Na Figura 1 apresentamos duas imagens obtidas pelas buscas na internet.

É importante destacar que muitos alunos não reconhecem que a parábola é uma curva simétrica, ou seja, possui um eixo de simetria que passa pelo seu vértice. Logo, em muitos casos, confundem com semicírculos ou com outras curvas que não apresentam nenhuma simetria, conforme podemos observar nas imagens retiradas dos trabalhos dos alunos (Figura 2).

Neste sentido, Machado (2007) afirma ser preciso que os estudantes observem que as parábolas não são somente a representação gráfica da função quadrática, como geralmente são

\footnotetext{
3 Disponível em http://repositorio.unb.br/handle/10482/13603; acessado em 29 set. 2019.
} 
trabalhadas em sala de aula, de uma forma quase sem utilidade; é necessário explorar este conteúdo, abordando algumas aplicações das parábolas, mostrando que são essenciais na construção de objetos comuns no cotidiano, tornando este assunto mais significativo.
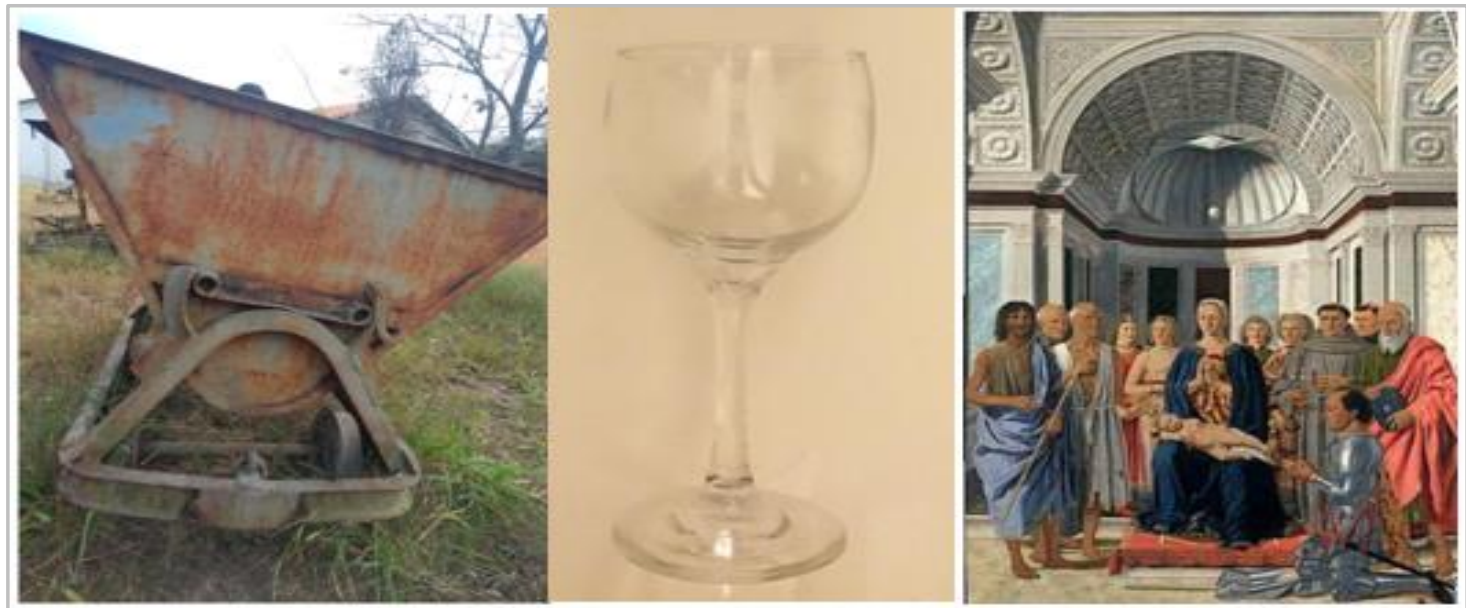

Figura 2: Imagens de formas de parábolas retiradas dos trabalhos dos estudantes (Acervo da Pesquisa)

Em seguida, cada grupo foi convidado a apresentar e explicar quais eram as imagens escolhidas. Ao final de todas as apresentações, os estudantes foram questionados se parte das imagens obtidas tinham a forma de parábolas e como poderiam comprovar suas respostas. Houve neste momento intenso debate e diversas opiniões foram apresentadas e acolhidas, ficando para a aula seguinte a comprovação. Para a próxima aula, segunda etapa da atividade, os estudantes foram orientados a fazer com seus smartphones no mínimo três registros fotográficos de imagens do cotidiano que, de alguma forma, remetesse à forma de parábola. Nesta etapa, esperava-se que eles buscassem os registros de antenas parabólicas, janelas, algumas pontes, luminárias etc., bem como fossem mais criteriosos na escolha das imagens, observando existência de vértice e simetrias em relação a este vértice, pois o professor já havia ensinado e construído os gráficos de algumas funções quadráticas e foram realizadas atividades de construção de gráfico com lápis e papel em folha quadriculada.

Quanto à representação gráfica de funções, Machado (2007) enfatiza que a elaboração de um gráfico por meio de simples transcrição de dados, tomados em uma tabela numérica, não permitirá ao estudante avançar na compreensão do comportamento das funções.

Na segunda etapa, após mostrarem seus registros, os estudantes foram desafiados a verificar se de fato as curvas por eles obtidas correspondiam a parábolas. Nesta ocasião, apesar dos conhecimentos que possuíam sobre função quadrática, tiveram dúvidas a respeito de como resolver o problema. Este tipo de dúvida é relatado na literatura e, conforme pesquisa de Heinen 
(2015), pode-se constatar que "os alunos não associam a lei da função quadrática com o seu gráfico" (p. 17).

Neste momento foi possível notar que, apesar dos referidos conhecimentos que os estudantes detinham, tiveram dúvidas e perceberam que precisariam investigar. Depois de pesquisarem utilizando seus smartphones, observaram que uma forma de resolver o problema era encontrando a expressão matemática da curva, ou seja, o modelo matemático que a representava. Esta também é uma das dificuldades relatadas por pesquisadores, pois não é muito usual partir da representação gráfica em busca da expressão algébrica que representa a parábola. Segundo Maia (2007), nos livros didáticos

há a predominância de duas formas da passagem da representação algébrica para a representação gráfica: por meio da construção de tabelas, que na maioria das vezes são escolhidos números inteiros, ou utilizando-se alguns pontos especiais, aos quais os autores dos livros chamam de pontos notáveis da parábola (p. 55).

Essa autora observa que a passagem inversa, ou seja, do gráfico para a expressão algébrica, pouco é realizada.

Nesta atividade, para obter a expressão algébrica, os estudantes utilizaram o aplicativo GeoGebra, pois o objetivo era encontrar o modelo matemático que representava a curva, sendo que a passagem da representação gráfica para a algébrica seria abordada com mais detalhes no desenrolar do curso. Munidos dessas informações, utilizando os recursos de seus smartphones, inseriram suas imagens no aplicativo mencionado e, manuseando as ferramentas dele, investigaram as curvas, discutiram com seus pares e concluíram se as imagens representavam ou não uma parábola (Figura 3).

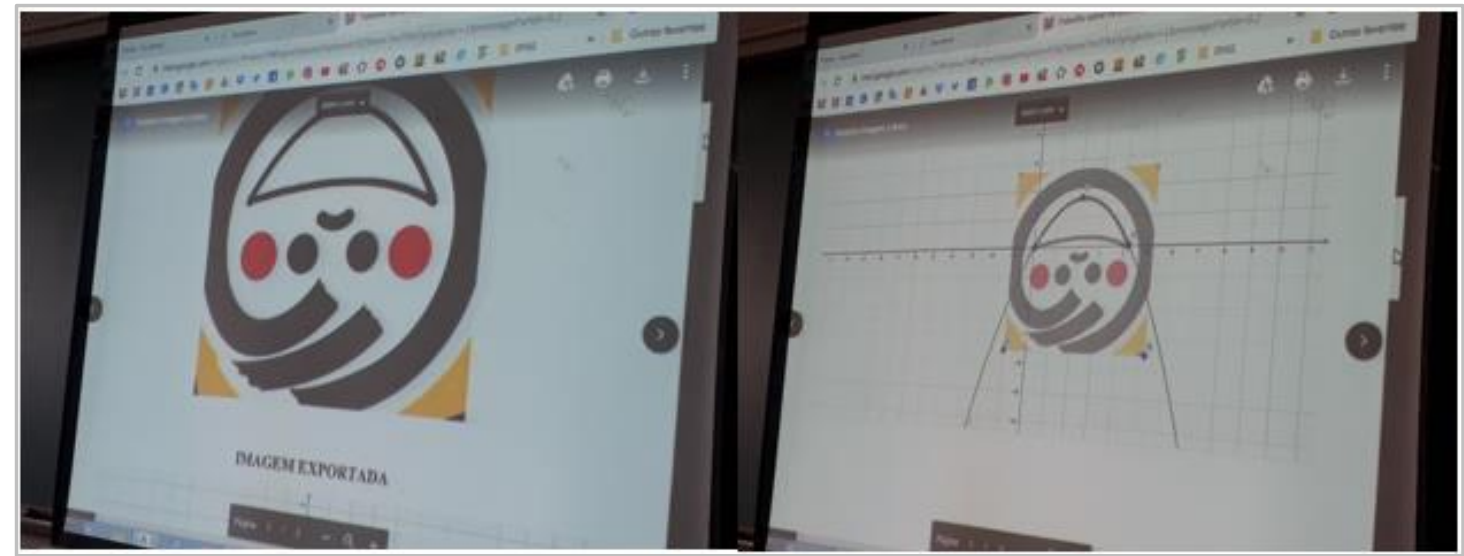

Figura 3: Imagem do momento de comprovação das curvas (Acervo da Pesquisa) 
$\mathrm{Na}$ terceira etapa, os estudantes foram desafiados a determinar nas imagens das estruturas fotografadas trazidas por eles, pontos importantes e o que representavam no contexto real. Ou seja, o que simbolizavam as alturas, larguras e distâncias na imagem em relação à estrutura física apresentada (Figura 4).

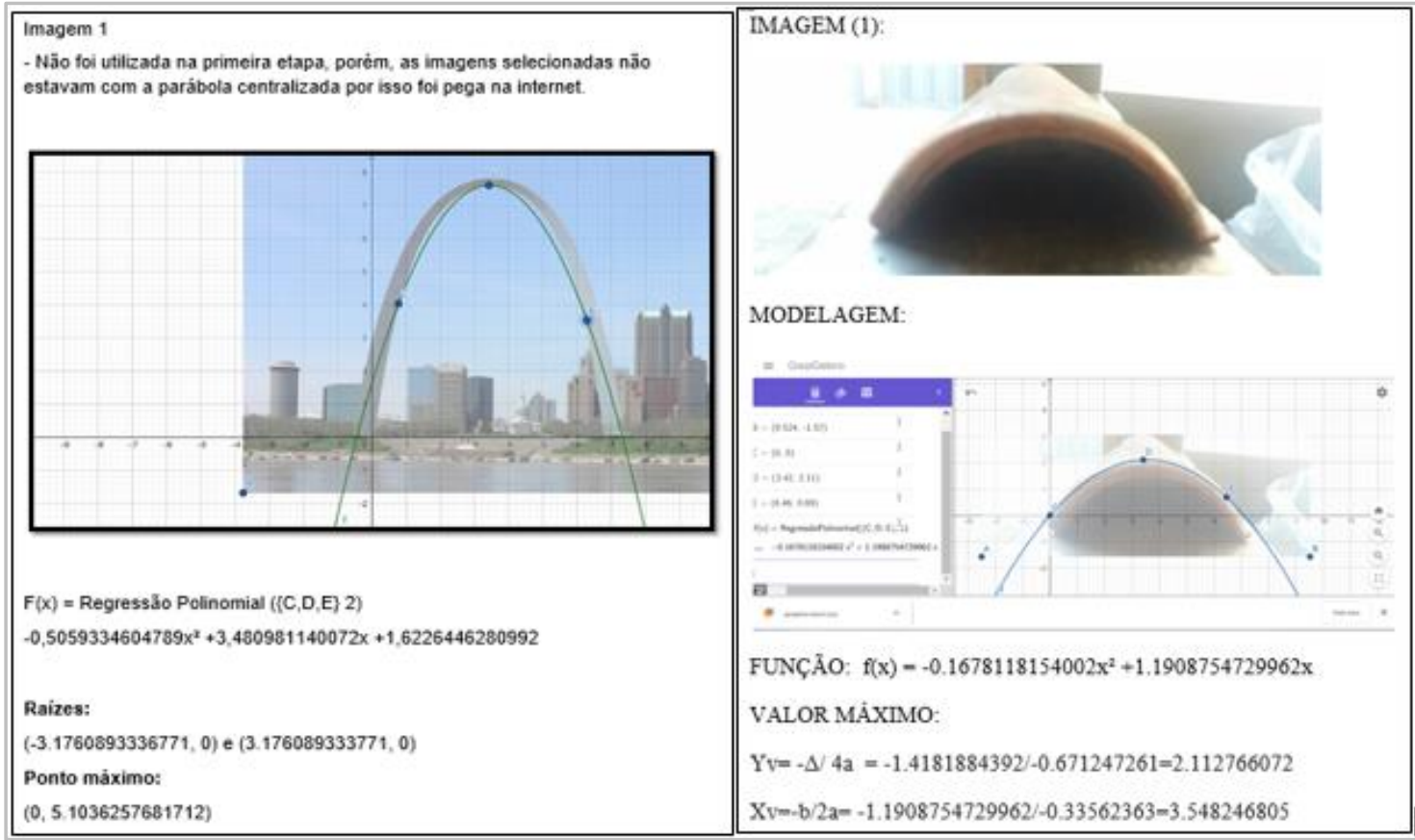

Figura 4: Imagem da modelagem realizada pelos alunos (Acervo da Pesquisa)

Sendo assim, investigaram o vértice da parábola (em caso afirmativo da curva) para estimar o valor máximo ou mínimo (alturas) e as raízes (distâncias horizontais). O objetivo dessa etapa da atividade foi mostrar para os estudantes sobre a importância do vértice e sua aplicação em relação aos valores máximos ou mínimos atribuídos aos pontos da imagem da função. Segundo Maia (2007), os estudantes têm dificuldades em enxergar o gráfico como um todo quando não utilizam um software ou aplicativo e tentam sempre aplicar o que estão aprendendo na aula regular, ou seja, buscam pelos pontos notáveis que são muito abordados durantes as exposições em sala. Portanto eles aprendem e sabem calcular as coordenadas destes pontos, mas não conseguem interpretar sua utilização em problemas de aplicação. Em relação às imagens apresentadas, foi debatido com os estudantes o que representavam os pontos calculados e como interpretá-los numa situação real.

Durante as etapas da atividade, aconteceram momentos de aprendizado com mobilidade, Mobile Learning; o conhecimento foi proporcionado pela utilização dos smartphones e seus aplicativos nas ocasiões de interação na sala de aula e nos ambientes virtuais. As dúvidas e 
sugestões foram inteiramente compartilhadas no grupo de WhatsApp de cada turma. Para avaliar a participação dos estudantes, foi solicitado aos grupos que todos os procedimentos durante o uso do aplicativo para obter o modelo, bem com medir, calcular e estimar os valores solicitados em relação ao modelo encontrado (equação da curva) fossem filmados e devidamente explicados para serem disponibilizados aos demais colegas da turma e ao professor.

Ao final, os grupos entregaram um relatório da atividade contendo: suas percepções em relação à atividade desenvolvida, as figuras investigadas, a imagem da modelagem realizada com o aplicativo (Figura 4), a equação da curva (modelo matemático), os cálculos algébricos confirmando as coordenadas do vértice e suas conclusões. Este material foi avaliado pelo professor e compôs a nota do aluno.

\section{Análise da abordagem e das atividades desenvolvidas}

Nesta seção será apresentada uma análise de cunho predominantemente qualitativo da abordagem e das tarefas desenvolvidas, tendo como norte os referenciais teóricos e a questão de investigação que balizaram a pesquisa. Sendo assim, foi elaborado o Quadro 1, que alinha a atividade desenvolvida com as estratégias utilizadas, tomando como referência os objetivos da atividade, as competências e habilidades a serem desenvolvidas, os papéis da Modelagem Matemática e do Mobile Learning, os resultados e evidências.

Quadro 1: Aspectos das atividades desenvolvidas

\begin{tabular}{|l|l|}
\hline \multicolumn{1}{|c|}{} & \multicolumn{1}{c|}{ Parábolas na Arquitetura } \\
\hline Objetivos iniciais & $\begin{array}{l}\text { Construir um entendimento matemático a partir do conhecimento e das } \\
\text { experiências informais dos estudantes. } \\
\text { Promover iniciação dos estudantes à Modelagem Matemática e proporcionar, no } \\
\text { segundo momento, familiarização com a temática. } \\
\text { Desenvolver a competência de Modelagem Matemática (BLUM e LEIß, 2007). } \\
\text { Desenvolver as habilidades e competências essenciais e específicas da BNCC } \\
\text { (BRASIL, 2017). } \\
\text { Construir situações para contextualizar o estudo de função quadrática. } \\
\text { Construir, ler e analisar gráficos de funções polinomiais do } 2^{\circ} \text { grau com o auxilio } \\
\text { de tecnologia digital móvel (aplicativo GeoGebra). } \\
\text { Resolver problemas envolvendo máximos (ou mínimos) da função quadrática, } \\
\text { relacionando-os também à Geometria e com sua aplicação em contextos reais. }\end{array}$ \\
\hline Competências e & CG1: "Valorizar e utilizar os conhecimentos construídos [...] para entender e \\
\hline
\end{tabular}




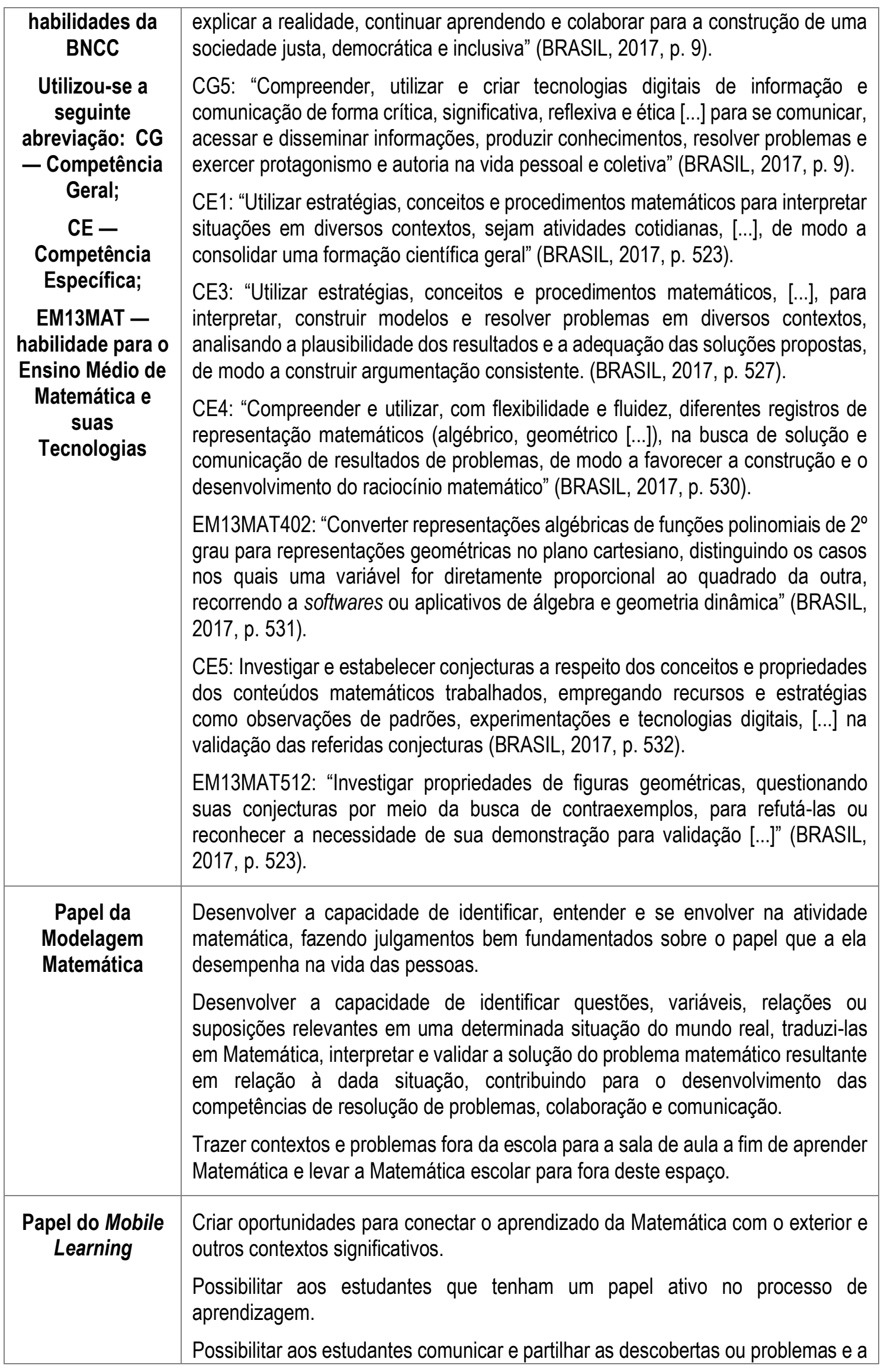




\begin{tabular}{|l|l|}
\hline & $\begin{array}{l}\text { captação de dados e informações para serem usados em suas investigações. } \\
\text { Proporcionar o desenvolvimento de trabalho individual e colaborativo. } \\
\text { Proporcionar experimentação usando a Tecnologia. } \\
\text { Possibilitar a entrega de conteúdos de aprendizagem de forma rápida em qualquer } \\
\text { lugar ou momento. } \\
\text { Permitir criar um ambiente mais personalizado e dinâmico de aprendizagem. }\end{array}$ \\
\hline Resultados e & $\begin{array}{l}\text { Os estudantes ao utilizarem seus smartphones, compreenderam que as } \\
\text { tecnologias digitais de informação e comunicação podem contribuir para o } \\
\text { aprendizado de Matemática. } \\
\text { Os estudantes mostraram habilidades voltadas às capacidades de investigação e } \\
\text { de formulação de explicações e argumentações que emergiram da experiência } \\
\text { proporcionada pela modelagem com o apoio visual de seus smartphones. } \\
\text { Os estudantes buscaram imagens, manipularam e investigaram seus } \\
\text { comportamentos, verificando se as curvas obtidas possuíam características de } \\
\text { função quadrática (modelo matemático). } \\
\text { Produziram gráficos de funções quadrática utilizando o aplicativo GeoGebra. } \\
\text { Os estudantes avaliaram se os seus gráficos foram construídos de forma correta, } \\
\text { observando suas concavidades e suas interseções com os eixos. } \\
\text { Os estudantes avaliaram os pontos de interseção com os eixos e o ponto extremo } \\
\text { da função quadrática, analisaram a relação existente entre estes pontos e os } \\
\text { coeficientes da expressão algébrica utilizando o GeoGebra. } \\
\text { Os estudantes elaboraram o relatório da atividade em que descrevem estratégias, } \\
\text { conceitos e procedimentos matemáticos para interpretar a situação proposta. } \\
\text { Os estudantes escreveram e relataram suas observaçães e suas percepções. } \\
\text { Os estudantes produziram conjecturas sobre a utilização e aplicação das funções } \\
\text { quadráticas nas estruturas construídas pelo homem existentes nas cidades. } \\
\text { Os estudantes desenvolveram e aplicaram um modelo matemático para solucionar } \\
\text { o problema e validaram-no junto à turma, apoiados em argumentos bem } \\
\text { fundamentados. } \\
\text { Houve resultados positivos das atividades resolvidas e entregues pelos alunos } \\
\text { durante as aulas, bem como aproveitamento satisfatório no teste individual } \\
\text { aplicado. }\end{array}$ \\
\hline
\end{tabular}

Fonte: Elaboração dos Autores

Conforme pode ser observado no Quadro 1, a estratégia de utilizar os dispositivos móveis (smartphones) e Modelagem Matemática na atividade trouxe contribuições para 0 desenvolvimento de habilidades e competências, conforme propõem a BNCC (BRASIL, 2017) e a publicação da UNESCO (2016). As colaborações puderam ser observadas nos relatos dos estudantes, nos dados coletados nas explicações, nas apresentações e nos trabalhos, conforme mostraremos e descreveremos a seguir. 
A primeira contribuição está relacionada com o papel da Matemática na vida das pessoas. Isso pode ser percebido quando observamos o desenvolvimento e as estratégias dos estudantes para resolver as situações colocadas, ao propor um modelo matemático para solucionar 0 problema, buscando imagens, manipulando e investigando seus comportamentos, verificando se possuem características de função quadrática e opinando se as curvas podem ser representadas por um modelo matemático.

Ademais, podemos afirmar, também, que as atividades realizadas contribuíram para desenvolver o conhecimento matemático. De fato, as tarefas possibilitaram aos estudantes aprimorar a capacidade de identificar, entender e se envolver na atividade matemática, fazendo julgamentos fundamentados sobre o papel que a Matemática desempenha na vida das pessoas e da sociedade (OCDE, 2006).

Também entendemos que a atividade contribuiu para iniciar os estudantes na abordagem da Modelagem Matemática, bem como desenvolver esta competência, conforme Blum e Leiß (2007), uma vez que a atividade levou os estudantes a desenvolver sua capacidade de identificar questões, variáveis, relações ou suposições relevantes sobre as situações do mundo real investigadas; traduzi-las em Matemática; interpretar e validar a solução do problema matemático resultante de cada situação, contribuindo para o desenvolvimento das competências de resolução de problemas, colaboração, pensamento crítico e comunicação.

Analisando os dados, constatou-se que a atividade de modelagem contribuiu para aprimorar algumas habilidades e competências como orienta a BNCC. Ao observarmos as falas dos estudantes durante as explicações e apresentações dos grupos, foi possível perceber contribuições para o desenvolvimento da competência geral CG1, pois os estudantes puderam valorizar e utilizar os conhecimentos construídos para entender e explicar a realidade, continuar aprendendo e colaborar para a construção de uma sociedade justa, democrática e inclusiva.

Em relação ao avanço de conteúdo, a atividade proporcionou um contexto real para desenvolver os conceitos relacionados à teoria das funções quadráticas, contribuindo para adquirir as competências específicas 1 e 3 da BNCC.

Neste sentido, podemos perceber na forma como os estudantes utilizaram estratégias, conceitos e procedimentos matemáticos para interpretar como as parábolas, nos diversos contextos nas quais as imagens foram obtidas, contribuíram para consolidar o conhecimento em relação a este tipo de função polinomial. Colaboraram também para 0 entendimento e 
interpretação da representação gráfica da função quadrática, construção de modelos, representação algébrica e para a resolução do problema colocado, analisando a plausibilidade dos resultados e a adequação das soluções propostas, de modo a construir argumentação consistente. (BRASIL, 2017).

Ao investigarem com o auxílio dos smartphones, imagens de parábolas na web, na cidade e nos objetos de seu cotidiano, buscando dados e informações para fazer conjecturas sobre a representação de uma função quadrática, os estudantes desenvolveram também a competência geral CG5, pois compreenderam e utilizaram as "tecnologias digitais de informação e comunicação de forma crítica, significativa, reflexiva e ética [...] para se comunicar, acessar e disseminar informações, produzir conhecimentos, resolver problemas e exercer protagonismo e autoria na vida pessoal e coletiva" (BRASIL, 2017, p. 9).

Ao utilizar o aplicativo GeoGebra para analisar as representações gráficas e algébricas das imagens, foi possível observar contribuições para desenvolvimento da competência específica CE4, já que os estudantes compreenderam e realizaram, com flexibilidade e fluidez, os diferentes registros de representação matemáticos (algébrico, geométrico), buscando solucionar o problema, apresentando os resultados e conclusões, construindo o desenvolvimento do raciocínio matemático. Consequentemente, a habilidade de converter representações algébricas de funções polinomiais de $2^{\circ}$ grau para representações geométricas no plano cartesiano também foi aprimorada, contribuindo, assim, para o desenvolvimento da habilidade EM13MAT402.

Também foi possível observar que houve contribuição para o desenvolvimento da competência específica CE5, pois possibilitou aos estudantes investigar e estabelecer conjecturas a respeito do conceito e propriedades das funções quadráticas por meio da utilização do aplicativo GeoGebra e da Modelagem para observações de padrões, experimentações e validação das referidas conjecturas, ao investigar as propriedades geométricas da parábola, questionando suas conjecturas por meio da busca de contraexemplos, imagens que não representavam parábolas, para refutá-las ou reconhecer a necessidade de demonstrá-las por meio da expressão algébrica para validar suas conclusões, eles desenvolveram a habilidade EM13MAT512.

Ainda em relação ao conhecimento matemático, a atividade contribuiu para que os estudantes mobilizassem seu modo próprio de raciocinar, representar, argumentar e comunicar de forma alinhada com as orientações da BNCC. As competências que envolvem o ato de raciocinar foram desenvolvidas quando os estudantes investigaram, explicaram e justificaram os 
problemas resolvidos com ênfase nos processos de argumentação matemática. As competências diretamente associadas a representar foram desenvolvidas na elaboração de registros para evocar um objeto matemático. Já a referente à comunicação foi desenvolvida nas apresentações, justificativas dos resultados encontrados e nas interações com os colegas. Por fim, a competência de argumentar pôde ser desenvolvida na formulação e testagem das conjecturas que ocorreram nas apresentações e justificativas do modelo matemático proposto.

\section{Considerações}

Neste artigo, apresentamos uma investigação que apontou contributos da utilização de atividades de Modelagem Matemática, tendo como suporte os dispositivos móveis (smartphones) para o desenvolvimento de conteúdos e competências gerais e específicas relacionadas com 0 ensino de Matemática no Ensino Médio.

A partir das análises dos dados e dos resultados de aprendizagem obtidos na atividade relatada, foi possível perceber que 0 ambiente proporcionado pelas metodologias contribuiu para o ensino do conteúdo matemático relacionado com os conceitos de função quadrática, ao possibilitar uma abordagem contextualizada destes conceitos, mostrando sua aplicação, tornando 0 assunto mais significativo e facilitando a assimilação do conteúdo.

Em relação às contribuições para o desenvolvimento de competências, conforme orientações da BNCC e de publicações da Unesco, foi possível perceber que o ambiente de aprendizagem auxiliou na promoção da competência de modelagem e para o desenvolvimento de algumas competências gerais, relacionadas com o conhecimento matemático e cultura digital. Contribuiu também para o desenvolvimento de competências específicas da área de Matemática, relacionadas com a utilização de estratégias, conceitos e procedimentos matemáticos para interpretar, construir modelos e resolver problemas, analisando a plausibilidade dos resultados e a adequação das soluções propostas, de modo a construir argumentação consistente. Por fim, contribuiu para o desenvolvimento das habilidades de conversão de representações algébricas e geométricas das funções quadráticas, utilizando o aplicativo de álgebra e geometria dinâmica, 0 que possibilitou aos estudantes investigar propriedades destas funções, questionar suas conjecturas por meio da busca de contraexemplos, para refutar ou reconhecer se as curvas e seus modelos representavam uma parábola. Contribuiu para ajudar os estudantes a entender por quê o conhecimento matemático e as habilidades de modelar e resolver problemas serão úteis no seu 
processo de aprendizagem.

A estratégia de utilizar o smartphone e os aplicativos trouxe contribuições para o ambiente de aprendizagem por possibilitar aos estudantes disponibilizar informações que foram coletadas durante a troca de mensagens com seus colegas e professores, mostrando suas habilidades e dificuldades quando testavam hipóteses, percebiam lacunas em seu conhecimento e engajavamse em análises dos conteúdos abordados. Também possibilitou ao professor receber e comunicar informações, entregar material de estudo, interagir para sanar dúvidas e propor soluções no exato momento que o estudante precisou ou solicitou. Foi possível observar que os aplicativos foram utilizados no contexto educativo para mandar mensagens de texto, áudios, imagens etc., possibilitando, assim, ao professor estender a educação além dos limites físicos da sala de aula e além dos períodos fixos do dia escolar, contribuindo para a aprendizagem com mobilidade. Ademais contribuiu para estimular os estudantes a participar de forma mais ativa da sua própria aprendizagem.

Por fim, entendemos ser pertinente a investigação para fomentar o debate sobre a integração da Modelagem Matemática e do Mobile Learning. Estas tendências, conforme mostrou a investigação, podem contribuir para os processos de ensino e de aprendizagem da Matemática no Ensino Médio. No entanto, acreditamos que seja preciso mais pesquisas para investigar a melhor forma de realizar esta integração.

\section{Referências}

ALMEIDA, Lourdes Maria Werle de; DIAS, Michele Regiane. Um estudo sobre o uso da Modelagem Matemática como estratégia de ensino e aprendizagem. Bolema, Rio Claro, v. 17, n. 22, p. 19-35, set. 2004.

ALMEIDA, Lourdes Maria Werle de; SILVA, Karina Pessoa; VERTUAN, Rodolfo Eduardo. Modelagem Matemática na Educação Básica. São Paulo: Contexto, 2012.

ARAÚJO JUNIOR, Carlos Fernando de; DIAS, Eduardo Jesus; CONTI, Carmen Lúcia Tozzi Mendonça; OTA, Marcos André. Tendências do m-learning na Educação Básica e o desenvolvimento de competências para o século 21. REnCiMa, São Paulo, v. 10, n. 4, p. 181-191, 2019.

BARBOSA, Jonei Cerqueira. A "contextualização" e a Modelagem na Educação Matemática do Ensino Médio. In: ENCONTRO NACIONAL DE EDUCAÇÃO MATEMÁTICA, 8, 2004, Recife. Anais do VIII ENEM. Recife: SBEM, 2004, p. 1-8.

BASSANEZI, Rodney Carlos. Ensino-aprendizagem com Modelagem Matemática: uma nova 
estratégia. São Paulo: Contexto, 2002.

BLUM, Werner. Quality teaching of Mathematical Modelling: what do we know, what can we do? In: INTERNATIONAL CONGRESS ON MATHEMATICAL EDUCATION, 12, 2015, Seol. Proceedings of the ICME-12. Seol: ICME, 2015, p. 73-96.

BLUM, Werner; FERRI, Rita Borromeo. Mathematical Modelling: can it be taught and learnt? Journal of Mathematical Modelling and Aplication, Blumenau, v. 1, n. 1, p. 45-58, 2009.

BLUM, Werner; LEIß, Doninik. How do students and teachers deal with modelling problems? In: HAINES, Christopher; GALBRAITH, Peter; BLUM, Werner; KHAN, Sanowar. (Ed). Mathematical Modelling: Education, Engineering and Economics. England: Woodhead Publishing, 2007, p. 222231.

BOLZAN, Tiago Dias; FLORES, Maria Lúcia Pozzatti; GOI, Mara Elisângela Jappe. Ensino da função quadrática através da metodologia de resolução de problemas. 2014. 31f. Trabalho de Conclusão de Curso (Licenciatura em Ciências Exatas) Universidade Federal do Pampa. Caçapava do Sul.

BORBA, Marcelo de Carvalho; PENTEADO, Mirian Godoy. Informática e Educação Matemática. Belo Horizonte, Autêntica, 2010.

BRAGA, Roberta Modesto; ESPÍRITO SANTO, Adilson de Oliveira do. Modelagem Matemática na perspectiva da Teoria da Atividade de Engeström. Boletim do LABEM, Niteroi, v. 8, n. 14, p. 124142, jan./jun. 2017.

BRASIL. Ministério da Educação. Secretaria de Educação Básica. Base Nacional Comum Curricular. Brasília, MEC/SEB, 2017.

BRIZ-PONCE, Laura; PEREIRA, Anabela; CARVALHO, Lina, JUANES-MÉNDEZ, Juan Antônio; GARCÍA-PEÑALVO, Francisco José. Learning with mobile Technologies: students' behavior. Computers in Human Behavior, v. 72, p. 612-620, jul. 2017.

CROMPTON, Helen. A historical overview of M-Learning: toward learner-centered education. In: BERGE, Zane L.; MUILENBURG, Lin Y. (Ed.). Handbook of mobile learning. New York: Routledge, 2013, p. 41-52.

CROMPTON, Helen; TRAXLER, John. Introduction. In: CROMPTON, Helen; TRAXLER, John. (Ed.). Mobile Learning and Mathematics: fundamentals, design and case. New York: Routledge, 2015, p. 1-8.

D'AMBRÓSIO, Ubiratan. Da realidade à ação: reflexos sobre Educação e Matemática. São Paulo: Summus, 1986.

ENGLISH, Lyn. STEM: Challenges and opportunities for Mathematics Education. In: ANNUAL CONFERENCE OF THE INTERNATIONAL GROUP FOR THE PSYCHOLOGY OF MATHEMATICS EDUCATION, 39, 2015, Hobart. Proceedings of the PME 39. Hobart: International Mathematical Union, 2015, p. 4-18.

FERREIRA, Neuber Silva. Modelagem Matemática e Tecnologias de Informação e Comunicação 
como ambiente para abordagem do conceito de Função segundo a Educação Matemática Crítica. 2013. 243f. Dissertação (Mestrado em Educação Matemática) - Instituto de Ciências Exatas e Biológicas. Universidade Federal de Ouro Preto. Ouro Preto.

HEINEN, José Guilherme. Ensino de função quadrática utilizando o GeoGebra. 2015. $21 f$. Trabalho de Conclusão de Curso (Especialização em Matemática, Mídias Digitais e Didática para a Educação Básica) — Instituto de Matemática. Universidade Federal do Rio Grande do Sul. Porto Alegre.

MACHADO, Mirtes Tamy Gomes. Parábolas: as curvas preciosas. 2007. 20f. Monografia (Programa de Desenvolvimento Educacional) - Secretaria de Estada da Educação do Paraná; Universidade Estadual de Londrina. Londrina.

MAIA, Diana. Função Quadrática: um estudo didático de uma abordagem computacional. 2007. 141f. Dissertação (Mestrado em Educação Matemática) - Faculdade de Ciências Exatas e Tecnologias. Pontifícia Universidade Católica de São Paulo. São Paulo.

MOURA, Adelina Maria Carreiro. Apropriação do telemóvel como ferramenta de mediação em mobile Learning: estudos de caso no contexto educativo. 2010. 630f. Tese (Doutorado em Ciências da Educação) - Instituto de Educação. Universidade do Minho. Braga.

NUNES, José Manuel Freixo. Mobile learning e pensamento computacional: contributos para o desenvolvimento de aplicações em contextos educativos. 2019. 546f. Tese (Doutorado em Educação). Universidade Aberta de Portugal. Lisboa.

OCDE - ORGANIZAÇÃO PARA A COOPERAÇÃO E DESENVOLVIMENTO ECONÔMICO. Compétences en Sciences, Lecture et Mathématiques: le cadre d'évaluation de PISA 2006. Paris: OCDE, 2006.

OCDE - ORGANIZAÇÃO PARA A COOPERAÇÃO E DESENVOLVIMENTO ECONÔMICO. PISA 2012 assessment and analytical framework: Mathematics, Reading, Science, Problem Solving and Financial Literacy. Whashington: OCDE, 2013.

PÁDUA, Mário Cesar Daldegan. Utilizando o software GeoGebra como ferramenta auxiliar no ensino de função afim e função quadrática. 2011. 34f. Monografia (Unidade do Plano Integrado de Formação Continuada do Programa de Desenvolvimento). Secretaria de Estado da Educação do Paraná; Universidade Tecnológica Federal do Paraná. Curitiba.

PEREIRA, Rudolph dos Santos Gomes; SEKI, Jeferson Takeo Padoan; PALHARINI, Bárbara Nivalda; NETO, João Coelho; SILVA, Ariel Cardoso da; DAMIN, Willian; MARTINS, Bianca de Oliveira. Modelagem Matemática e tecnologias digitais educacionais: possibilidades e aproximações por meio de uma revisão sistemática de literatura. REnCiMa, São Paulo, v. 8, n. 2, p. 80-94, 2017.

PLOMP, Tjeerd; NIEVEEN, Nienke. Educational design research part A: an introduction. Enchede: SLO, 2013.

RAMOS, Paula; GIANELLA, Taís Rabetti; STRUCHINER, Mirian. A pesquisa baseada em design em artigos científicos sobre o uso de ambientes de aprendizagem mediados pelas tecnologias da informação e da comunicação no ensino de Ciências. Alexandria, Santa Catarina, v. 3, n. 1, p. 77 - 
102, maio. 2010.

SÁ, Patrícia; PAIXÃO, Fátima. Competências-chave para todos no Séc. XXI: orientações emergentes do contexto Europeu. Interações, Santarém, v. 11, n. 39, p. 243-254, 2015.

SAWAYA, Sandra; PUTNAM, Ralph. Using mobile devices to connect Mathematics to out-ofschool contexts. In: CROMPTON, Helen; TRAXLER, John. (Ed.). Mobile Learning and Mathematics: fundamentals, design and case. New York: Routledge, 2015, p. 9-19.

SILVA, Cintia da. Aprendizagem Significativa em atividades de Modelagem Matemática, 2018. 145f. Tese (Doutorado em Ensino de Ciências e Educação Matemática) - Centro de Ciências Exatas. Universidade Estadual de Londrina. Londrina.

UNESCO — ORGANIZAÇÃO DAS NAÇÕES UNIDAS PARA A EDUCAÇÃO, A CIÊNCIA E A CULTURA. O Futuro da aprendizagem móvel: implicações para planejadores e gestores de políticas. Brasília: Unesco, 2014.

UNESCO — ORGANIZAÇÃO DAS NAÇÕES UNIDAS PARA A EDUCAÇÃO, A CIÊNCIA E A CULTURA. Os desafios do ensino de Matemática na Educação Básica. Brasília: UNESCO; São Carlos: EdUFSCar, 2016.

VERTUAN, Rodolfo Eduardo. Um olhar sobre a Modelagem Matemática à luz da teoria dos registros de representação semiótica. 2007. 142f. Dissertação (Mestrado em Ensino de Ciências e Educação Matemática) - Centro de Ciências Exatas. Universidade Estadual de Londrina. Londrina.

ZANELLA, Marli Schmitt; KATO, Lilian Akemi. O desenvolvimento de competências a partir da modelagem matemática: um estudo com alunos da quarta série da escola primária alemã. Educere et Educare, Cascavel, v. 12, n. 24, p. 1-15, jan./abr. 2017. 